\title{
Glycemic Control Patterns and Kidney Disease Progression among Primary Care Patients with Diabetes Mellitus
}

\author{
Doyle M. Cummings, PharmD, Lars C. Larsen, MD, Lisa Doherty, MD, MPH, \\ C. Suzanne Lea, PhD, and Don Holbert, PhD
}

Background: Reducing glycosylated hemoglobin (HbA1c) to near or less than $7 \%$ in patients with diabetes is associated with diminished microvascular complications, but this level is not consistently achieved. The purpose of this study was to examine the relationship between fluctuations in HbA1c and changes in estimated glomerular filtration rate (eGFR) and estimated stage of chronic kidney disease (CKD) in an academic primary care practice.

Methods: We analyzed data from 791 diabetic primary care patients (25\% white; $75 \%$ African American) enrolled between 1998 to 2002 and followed through 2008 (mean follow-up, $7.6 \pm 1.9$ years). We calculated baseline and final follow-up eGFR using the Modification of Diet in Renal Disease equation. We examined the relationship between fluctuations in HbA1c and changes in eGFR and stage of CKD using multivariable linear and logistic regression models that controlled for demographic and clinical variables associated with CKD progression.

Results: From baseline to follow-up, mean eGFR in African Americans declined to a greater extent and more rapidly than in whites. Age, mean systolic blood pressure, initial HbA1c, initial eGFR, and number of HbA1c values (all $P<.01$ ) were significant predictors of change in eGFR. Among HbA1c fluctuation measures, the strongest predictor of change in eGFR was the proportion of HbA1c values $>7 \%$ $(P=.02)$; however, this contributed little to explaining model variance.

Conclusion: These data suggest that traditional demographic and clinical risk factors remain significantly associated with changes in eGFR and that the pattern of variability in HbA1c is only modestly important in contributing to changes in eGFR among African-American and white diabetic patients in primary care.(J Am Board Fam Med 2011;24:391-398.)

Keywords: Diabetes, Glycemic Control, Kidney Disorders

Prospective clinical trials have demonstrated that improvements in glycemic control, particularly early in treatment, are associated with reductions in the incidence of microvascular complications, in-

This article was externally peer reviewed.

Submitted 11 August 2010; revised 21 February 2011; accepted 3 March 2011.

From the Departments of Family Medicine (DMC, LCL, LD), Pediatrics (DMC), Public Health (DMC, CSL), and Biostatistics (DH), East Carolina University, Brody School of Medicine, Greenville, NC.

Funding: This research was supported in part by Health Resources and Services Administration grant no. D54HP03396.

Conflict of interest: none declared.

Corresponding author: Doyle M. Cummings, Brody School of Medicine, East Carolina University, 600 Moye Boulevard, Greenville, NC 27834 (E-mail: cummingsd@ecu.edu). cluding chronic kidney disease (CKD). ${ }^{1-9}$ Notably, the Diabetes Control and Complications Trial (DCCT) showed a significant relationship between reduction in glycosylated hemoglobin (HbA1c) levels and the risk of microvascular complications including CKD. ${ }^{1,2,9}$ Similar results were evident in the United Kingdom Prospective Diabetes Study, ${ }^{3,6}$ the Atherosclerosis Risk in Communities $^{7}$ study, and very recently in the Action to Control Cardiovascular Risk in Diabetes trial, ${ }^{8}$ with more profound hyperglycemia being associated with a greater risk of CKD. There seemed to be no threshold value of HbAlc above or below which complications were not increased or decreased, suggesting a continuous relationship between HbA1c and complications across a wide 
range of values. Long-term follow-up of patients in the DCCT and United Kingdom Prospective Diabetes studies demonstrated that glycemic control that improved early and was maintained for the study duration, even when lost at the end of the randomized trial study period, was associated with a reduction in the incidence of CKD after 10 years of follow-up. ${ }^{2,4}$ Furthermore, in a re-analysis of DCCT follow-up data, Lachin et $\mathrm{al}^{9}$ concluded that glycemic control, as measured by HbA1c, explained virtually all the difference in the risk of complications between intensive and conventional treatment groups. These findings suggest that the timing, magnitude, and duration of improvement in glycemic control among diabetic patients are important in the progression to CKD. In most prospective clinical trials evaluating diabetes outcomes such as the onset or progression of CKD, 2 groups with differing target levels of glycemic control (ie, $\mathrm{HbA1c}$ ) have been compared (eg, intensive treatment vs usual care). ${ }^{8}$ However, for longer-term studies, single measures or beginning and follow-up levels of HbA1c may inadequately characterize the pattern of glycemic control over the study period. In routine outpatient practice many diabetic patients do not consistently achieve the recommended level of glycemic control as defined by the American Diabetes Association. ${ }^{10}$ Large intra- and interindividual differences exist in the pattern of glycemic control. The causes of this variability are probably multifactorial and include diet, physical activity, medical care, access to medications, and medication adherence as well as insulin secretion and resistance. ${ }^{10}$

Some studies have averaged HbA1c values over the period of the study. We sought to expand on this concept by using additional mathematical techniques that take into consideration both the maximal value and extrapolated duration of abnormal HbA1c measures, including slope and area under and around the HbAlc versus time curve. Though these strategies have their own inherent limitations and assumptions (see Discussion), the intent of this approach was to provide a more precise characterization of the pattern of glycemic control in the real world of primary care practice and to assess the relationship to change in estimated glomerular filtration rate (eGFR) and stage of CKD. To our knowledge, this is the first study in which these measures of variation in $\mathrm{HbAlc}$ have been used to examine the relationship between patterns of glycemic control and diabetic complications. Specifically, the purpose of this study was to examine the relationship between fluctuations in glycemic control (HbAlc) over time and changes in eGFR and estimated stage of chronic kidney disease (CKD).

\section{Methods}

This retrospective cohort study examined adult patients ( $>18$ years of age) with a medical recordestablished diagnosis of type 2 diabetes mellitus who had been seen in one of the primary care practices (ie, family medicine, internal medicine) at an academic medical center in the southeastern United States during the baseline time period 1998 to 2002. Follow-up visits through 2008 for those patients with at least 5 measurements of glycemic control (HbA1c) were evaluated. We chose 5 HbA1c values as a minimum number necessary to characterize the slope and area under the curve (AUC) measures (described in more detail, below). All patients' medical records were abstracted, and data including demographic characteristics (age, race, sex, insurance); duration of diabetes; height; weight; blood pressure (BP); glycemic control (HbA1c); and serum creatinine were collected. Information about proteinuria was not consistently available and was not included in this study. For glycemic control, a HbA1c target value of $\leq 7 \%$ was used, as recommended by the American Diabetes Association. ${ }^{10}$ The eGFRs at baseline and at the most recent follow-up visit were calculated via computer using the Modification of Diet in Renal Disease $($ MDRD) equation $(\mathrm{eGFR}=186 \times$ serum creatinine $[\mathrm{mg} / \mathrm{dL}]^{(-1.154)} \times$ age $\left[\right.$ years] ${ }^{(-0.203)} \times$ 1.212 [if black] $\times 0.742$ [if female]). The MDRD equation was chosen based on data by Rigalleau et $\mathrm{al}^{11}$ demonstrating its utility in diabetic patients. In addition, the eGFR values at baseline and at the most recent follow-up were used to categorize each participant's stage of CKD based on the categorization available from the National Kidney Foundation: stage $1, \geq 90 \mathrm{~mL} / \mathrm{min}$; stage 2,60 to $89 \mathrm{~mL} /$ $\mathrm{min}$; stage 3,30 to $59 \mathrm{~mL} / \mathrm{min}$; stage 4,15 to 29 $\mathrm{mL} / \mathrm{min}$; stage $5,<15 \mathrm{~mL} / \mathrm{min}$ or dialysis. ${ }^{12}$ Patterns of glycemic control during the follow-up period were characterized by computing the following 10 measures from each patient's HbA1c profile: Overall mean HbA1c

$$
\text { Change score (initial - final HbA1c) }
$$


Slope of trend line of HbA1c versus time

Proportion of HbA1c values above 7\%

Area under the HbAlc versus time curve but above the 7\% threshold (see Figure 1)

Area between HbA1c versus time curve and the $7 \%$ threshold (area around threshold including area both above and below the threshold; see Figure 1)

Area difference $=$ area above $7 \%$ and below HbA1c versus time curve minus area below 7\% and under the curve (see Figure 1)

Figure 1. Examples of measures for patterns of fluctuation in glycosylated hemoglobin levels. Area under the curve for glycosylated hemoglobin versus time above $7 \%(t o p)$, approximately $7 \%$ (center), and the area difference (bottom).
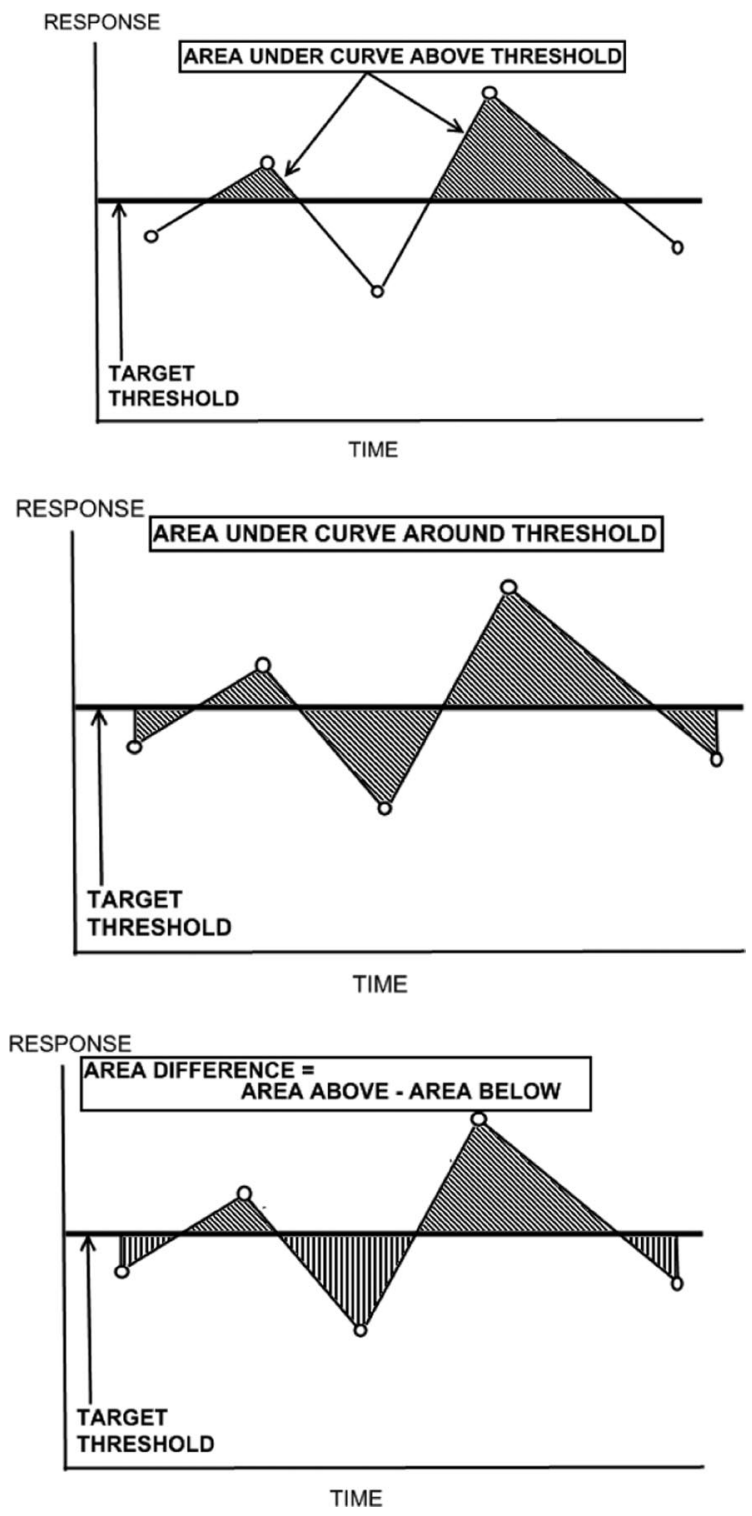

SD of HbA1c values: SD $=$ SQRT $([1 /\{n-1\}] \Sigma$ [value-mean] $]^{2}$ )

Root mean square of HbA1c values around 7\%: $\mathrm{rms}=\operatorname{SQRT}\left([1 / \mathrm{n}] \Sigma[\text { value }-7]^{2}\right)$

Average excess of HbA1c values above 7\%, averaged over all values exceeding $7 \%$

Examples of the measures involving area under the $\mathrm{HbA} 1 \mathrm{c}$ versus time curve that were calculated for each patient are graphically depicted in Figure 1. Correlations among the HbA1c profile measures and between the HbA1c profile measures and the demographic/clinical variables were examined. Stepwise linear regression modeling was then used to examine (1) the contribution of the demographic/ clinical variables to predicting the change in eGFR from baseline to final measurement, (2) the contribution of the HbA1c profile measures to predicting the change in eGFR from baseline to final measurement, and (3) the additional contribution of HbA1c measures while controlling for significant independent variables among demographic characteristics (age, race, sex) and clinical measures (duration of diabetes, BP, use of an angiotensin-converting enzyme inhibitor (ACEI) or angiotensin receptor blocker (ARB), initial eGFR, initial HbA1c, and number of HbA1c values). Logistic regression modeling was used to examine the contribution of these measures to predicting an increase of one or more CKD stages from baseline to final measurement while controlling for the same independent variables. The analysis was conducted using SAS software version 9.2 (SAS Institute, Cary, NC). Model parameters and 95\% CIs are reported.

\section{Results}

From a population of 2155 patients with diabetes who had at least one visit during the enrollment time period, a total of 791 patients who had at least $5 \mathrm{HbA1c}$ values over the follow-up period were included in the study. Mean age was $54 \pm 8$ years; $68 \%$ were women and $75 \%$ were African American. Baseline BP, HbA1c, and eGFR values and the percentage of use of ACEIs or ARBs by race and sex are given in Table 1, which also shows summary statistics for HbA1c decrease, eGFR decrease, and increasing stage of CKD. Mean follow-up was $7.6 \pm 1.9$ years; mean number of years from first to last $\mathrm{HbA1c}$ measurement was $6.78 \pm 2.0$ years; mean number of total primary 
Table 1. Mean Baseline and Change Statistics by Race and Sex

\begin{tabular}{|c|c|c|c|c|}
\hline Sex & Race & $\mathrm{n}$ & Variable* & Mean (SD) \\
\hline \multirow[t]{18}{*}{ Female } & \multirow[t]{9}{*}{ African American } & \multirow[t]{9}{*}{433} & Baseline SBP & $135.2(20.3)$ \\
\hline & & & Baseline DBP & $80.4(11.3)$ \\
\hline & & & Baseline HbA1c & $8.8(2.3)$ \\
\hline & & & Baseline eGFR & $100.6(30.7)$ \\
\hline & & & Taking ACEIs/ARBs (\%) & 47.6 \\
\hline & & & HbA1c decrease & $0.70(2.42)$ \\
\hline & & & eGFR decrease overall & $14.5(30.9)$ \\
\hline & & & Mean eGFR decrease/year & $1.9(4.2)$ \\
\hline & & & $\%$ worse CKD stage & 38.8 \\
\hline & \multirow[t]{9}{*}{ White } & \multirow[t]{9}{*}{102} & Baseline SBP & $129.5(18.0)$ \\
\hline & & & Baseline DBP & $75.6(11.0)$ \\
\hline & & & Baseline $\mathrm{HbA1c}$ & $7.5(1.9)$ \\
\hline & & & Baseline eGFR & $85.5(25.3)$ \\
\hline & & & Taking ACEIs/ARBs (\%) & 47.1 \\
\hline & & & HbA1c decrease & $0.50(2.10)$ \\
\hline & & & eGFR decrease overall & $6.5(25.1)$ \\
\hline & & & Mean eGFR decrease/year & $0.9(3.6)$ \\
\hline & & & $\%$ worse CKD stage & 35.0 \\
\hline \multirow[t]{18}{*}{ Male } & \multirow[t]{9}{*}{ African American } & \multirow[t]{9}{*}{162} & Baseline SBP & $133.2(20.4)$ \\
\hline & & & Baseline DBP & $80.1(12.1)$ \\
\hline & & & Baseline HbA1c & $8.9(2.5)$ \\
\hline & & & Baseline eGFR & $95.0(25.9)$ \\
\hline & & & Taking ACEIs/ARBs (\%) & 50.3 \\
\hline & & & HbA1c decrease & $0.96(3.15)$ \\
\hline & & & eGFR decrease overall & $12.0(32.7)$ \\
\hline & & & Mean eGFR decrease/year & $1.8(4.6)$ \\
\hline & & & $\%$ worse CKD stage & 36.9 \\
\hline & \multirow[t]{9}{*}{ White } & \multirow[t]{9}{*}{94} & Baseline SBP & $128.8(17.1)$ \\
\hline & & & Baseline DBP & $76.1(11.4)$ \\
\hline & & & Baseline HbA1c & $8.1(1.9)$ \\
\hline & & & Baseline eGFR & $91.5(24.7)$ \\
\hline & & & Taking ACEIs/ARBs (\%) & 43.0 \\
\hline & & & HbA1c decrease & $0.95(2.23)$ \\
\hline & & & eGFR decrease overall & $6.4(24.8)$ \\
\hline & & & Mean eGFR decrease/year & $1.0(3.8)$ \\
\hline & & & $\%$ worse CKD stage & 29.0 \\
\hline
\end{tabular}

SBP, systolic blood pressure; DBP, diastolic blood pressure; HbA1c, glycosolated hemoglobin; eGFR, estimated glomerular filtration rate; ACEIs, angiotensin converting enzyme inhibitors; ARBs, angiotensin receptor blockers; CKD, chronic kidney disease.

care visits was $57.96 \pm 33.14$ visits; mean number of serum creatinine measurements was $14 \pm 9.7$ measurements; and mean number of HbA1c measurements was $29.4 \pm 7.2$ measurements. Overall, there was a similar pattern of decline in eGFR from baseline to the end of follow-up, with African Americans of both sexes having a greater and more rapid decline (mean decline in eGFR, $12.0 \mathrm{~mL} / \mathrm{min} / 1.73 \mathrm{~m}^{2}$ for African-American men vs $6.4 \mathrm{~mL} / \mathrm{min} / 1.73 \mathrm{~m}^{2}$ for white men; $P=.12$; mean decline in eGFR, $14.5 \mathrm{~mL} / \mathrm{min} / 1.73 \mathrm{~m}^{2}$ for
African-American women vs $6.5 \mathrm{~mL} / \mathrm{min} / 1.73$ $\mathrm{m}^{2}$ for white women; $\left.P<.01\right)$ than that observed among white patients (see Figure 2).

\section{Correlation Analysis}

The $10 \mathrm{HbA1c}$ profile measures of glycemic control variability were highly correlated with one another. The highest intercorrelations (exceeding $0.9)$ were AUC above $7 \%$, with AUC approximately 7\% ( $\mathrm{r}=0.98)$, AUC difference $(\mathrm{r}=0.98)$, and $\mathrm{HbAlc}$ mean $(\mathrm{r}=0.91)$, as well as AUC dif- 
Figure 2. Decline in mean estimated glomerular filtration rate (GFR) by race and sex, 1998 to 2008 .

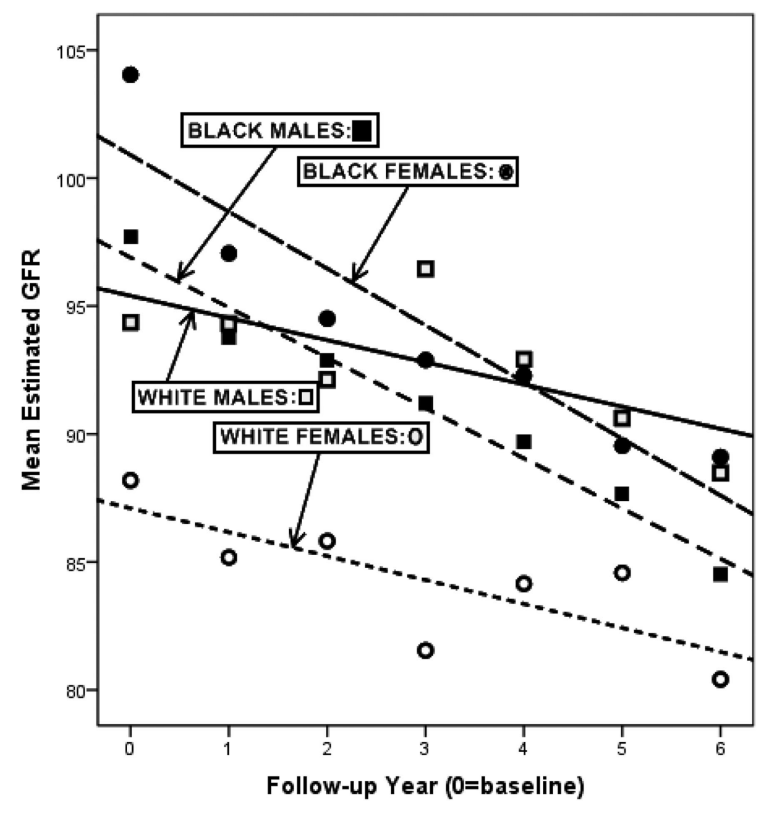

ference with HbAlc mean $(r=0.94)$ and AUC approximately $7 \%(\mathrm{r}=0.92)$.

Six of the correlations between HbAlc profile measures and demographic/clinical variables exceeded 0.5 , and all of these involved the baseline $\mathrm{HbA1c}$ value. Baseline HbA1c was correlated with HbA1c decrease $(r=0.65)$, HbA1c mean $(r=$ $0.58)$, average HbA1c excess above $7 \%(r=0.57)$, $\mathrm{HbA1c}$ root mean square approximately $7 \%(\mathrm{r}=$ $0.56)$, AUC difference $(\mathrm{r}=0.52)$, and AUC above $7 \%(\mathrm{r}=0.51)($ all $P<.01)$.

Decrease in eGFR was correlated with duration of diabetes $(\mathrm{r}=0.18 ; P<.01)$ and with the 3 area measures: AUC above HbAlc value of $7 \%(\mathrm{r}=$ $0.16 ; P<.01)$; AUC around HbA1c value of $7 \%$ $(\mathrm{r}=0.16 ; P<.01)$; and the area difference $(\mathrm{r}=$ $0.15 ; P<.01)$.

\section{Linear Regression Modeling}

Collectively, the 11 demographic/clinical variables explained $27.9 \%$ of the variation in eGFR decrease. Among these, the significant independent predictors of change in eGFR were age, mean SBP, initial $\mathrm{HbA1c}$, initial eGFR, and number of HbA1c values (all $P<.01$ ), but not race and sex. These 5 variables explained $27.3 \%$ of the variation in eGFR decrease.

By contrast, the HbA1c profile measures explained only $4.9 \%$ of the variation in eGFR decrease. To minimize confounding associated with multicollinearity, the AUC difference was omitted from the model because it was the least highly correlated with eGFR decrease. Those remaining HbA1c profile measures that made significant independent contributions in this revised model were AUC above 7\%, AUC approximately 7\%, HbA1c decrease, $\mathrm{HbA1c} \mathrm{SD}$, and $\mathrm{HbA1c}$ root mean square of approximately $7 \%$; collectively these 5 measures explained $4.5 \%$ of the variation in eGFR decrease.

To determine whether the HbA1c profile measures provided additional explanatory value beyond that provided by the significant demographic/clinical variables, a conditional stepwise regression analysis was conducted. The 5 significant demographic/clinical variables were forced into the model, whereas each $\mathrm{HbA1c}$ profile measure was added subsequently in stepwise fashion into the model. While controlling for the demographic/ clinical variables, among all measures characterizing glycemic control, the strongest predictor of change in eGFR was the proportion of HbA1c values exceeding $7 \%(P=.02)$. This stepwise addition increased the model $\mathrm{R}^{2}$ from $27.3 \%$ to $27.9 \%$, representing an improvement of only $2.2 \%$ in explaining variance. Regression model parameter estimates and confidence limits are shown in Table 2.

Table 2. Linear Regression Model of Significant Predictors of Change in Estimated Glomerular Filtration Rate

\begin{tabular}{lcc}
\hline Explanatory Variable & Fitted Coefficient & $95 \%$ Confidence Limits \\
\hline Age & 0.40 & $0.15,0.66$ \\
Baseline HbA1c & 1.63 & $0.73,2.53$ \\
Baseline estimated glomerular filtration rate & 0.47 & $0.40,0.54$ \\
HbA1c readings (n) & -0.81 & $-1.08,-0.55$ \\
Mean systolic blood pressure & 0.47 & $0.32,0.62$ \\
Proportion of HbA1c readings $>7 \%$ & -8.15 & $-13.64,-2.66$ \\
\hline
\end{tabular}

HbA1c, glycosolated hemoglobin. 
Table 3. Logistic Regression Model of Significant Predictors of Worsening by One or More Chronic Kidney Disease Stages

Odds Ratio

Explanatory Variable (95\% Confidence Limits)

Age

$1.037(1.009,1.056)$

Duration of diabetes in years

Mean systolic blood pressure

HbA1c decrease

Average excess of $\mathrm{HbAlc}>7 \%$

HbA1c, glycosolated hemoglobin.

\section{Logistic Regression Modeling}

For the logistic regression model, among demographic and clinical variables, age, mean SBP, and duration of diabetes (all $P<.05$ ) were the only significant predictors of worsening by one or more stage of CKD. While controlling for these variables, among all measures characterizing glycemic control, only 2 variables, namely the change in HbAlc (baseline minus final; $P=.01$ ) and the average excess of HbA1c above $7 \%(P=.01)$ provided significant additional explanatory power. Using the change in HbA1c from baseline to followup, we found that, for each 1-unit change in HbA1c, the odds of seeing a worsening in CKD stage increased by $8 \%$. Furthermore, for each 1 -unit increase in the average excess in HbAlc above $7 \%$, the odds of seeing a worsening in CKD stage increased by about $18 \%$. The odds ratios and confidence limits are given in Table 3.

\section{Discussion}

We used a novel approach to explore the association of patterns of $\mathrm{HbA1c}$ fluctuation with changes in eGFR and stage of CKD using medical record data from a population of diabetic patients seen in a primary care setting between 1998 and 2008. We demonstrated that the measureable effect of $\mathrm{HbA1c}$ fluctuations on progression of CKD over the study period, beyond the impact of routine demographic and clinical variables, was limited. This real-world observational study differs from controlled clinical trials in that our population, which was receiving routine primary care, demonstrated considerable fluctuations in HbA1c control relative to that observed in controlled clinical trials that study a more carefully selected group. Contrary to our expectation, these additional techniques only contributed modestly to our understanding of the relationship between glycemic control and change in eGFR and/or stage of kidney disease and were not superior to the simple baseline to final change in HbA1c.

Overall, study participants had only a modest decline in eGFR, consistent with expectations for our study population, which had a slightly younger mean age, a more limited duration of diabetes, and a significant percentage of patients who were being treated with ACEI/ARBs than the populations of some published trials. Our findings are consistent with previous literature in that we demonstrate that $\mathrm{BP}$ elevation, duration of diabetes, and age remain important predictors of change in eGFR. ${ }^{13-18}$ The identification of $\mathrm{BP}$ as a predictor of change in eGFR among our patients is important in that microvascular benefits from tight control of hypertension in patients with type 2 diabetes have been demonstrated after only 3 years of treatment, well within the 7.6-year median follow-up of patients in this study. ${ }^{14}$ Furthermore, a sustained reduction in blood pressure may be the single most important intervention in reducing the progression of $\mathrm{CKD}$ in patients with type 1 and type 2 diabetes. ${ }^{16}$ Our data reinforces the relationship between $\mathrm{BP}$ control and eGFR in these primary care patients.

Importantly, we also demonstrated a greater decline in eGFR among African Americans than that observed among whites, emphasizing the importance of this racial disparity. In our study, mean baseline systolic and diastolic BP as well as mean baseline HbA1c were higher in African Americans of both sexes, which may have contributed to the racial differences observed at follow-up. This suggests the need for additional tailored populationlevel strategies designed to improve risk factor management among African Americans with diabetes, as has been recommended in the National Kidney Foundation's clinical practice recommendations. ${ }^{17}$

The key finding of our work is that 5 variables, namely age, mean SBP, initial HbAlc, initial eGFR, and number of HbA1c values (all $P<.01$ ) explained $27.3 \%$ of the variation in change in eGFR; adding additional measures regarding HbA1c fluctuations explained an additional 2.2\% to $4.5 \%$ of the variation in eGFR decrease. Although their contribution to predicting changes in eGFR and worsening of CKD stage were less than the more established variables (eg, BP, duration of 
diabetes, age), the changes in these glycemic control pattern variables were at significant or nearsignificant levels and represent a contribution to our understanding.

This suggests that these strategies for estimating the pattern of fluctuations in glycemic control over time in primary care settings contribute only modestly to explaining the variance in change in eGFR and stage of CKD. This outcome has important implications. Clinicians often respond to fluctuations in $\mathrm{HbA1c}$ as independent events based on the understanding that tighter glycemic control will lead to greater reduction in the risk for microvascular complications. However, our findings and those of the recent re-analysis of the DCCT trial, ${ }^{9}$ while emphasizing the importance of HbAlc, suggest that small fluctuations in the pattern of HbAlc over time may be less important than the maximal change in HbA1c achieved with treatment. The concept of metabolic memory as suggested from the Epidemiology of Diabetes Interventions and Complications follow-up of DCCT participants, ${ }^{4}$ in which substantial reductions in $\mathrm{HbA1c}$ early on in the intensively treated group had long-lasting implications for reducing the risk of future microvascular complications, is consistent with this observation. Although speculative, this may imply that the pathophysiologic steps leading to microvascular complications such as CKD may not be associated with glycemic control (as measured by HbA1c) in a continuous fashion but perhaps through mechanisms that are more threshold driven and/or time dependent.

Though the contribution of HbAlc is significant, our findings underscore the importance of not considering $\mathrm{HbA1c}$ independently but rather in the context of other risk factors for CKD including BP, race, and age/duration of diabetes. Although the pattern of glycemic control contributed only modestly, and age, duration of diabetes, BP, and similar factors were independently predictive in the models studied, they collectively explained only $<30 \%$ of the variance in eGFR change. It is clear from this and other studies that other less well-described factors seem to explain the greatest variation in the risk of worsening eGFR. Active research programs are currently underway to identify new risk factors that may be more strongly and consistently linked to CKD. ${ }^{18}$
Treatment with ACEIs or ARBs of diabetic patients with confirmed microalbuminuria or proteinuria, even in the absence of hypertension, has been shown to slow progression of CKD. ${ }^{16,19}$ In our study, treatment with ACEIs/ARBs was not an independent predictor of change in eGFR or change in CKD stage, in part because the vast majority of patients were receiving these medications and this study was not designed to examine that question.

This study has a number of limitations. Assumptions inherent in extrapolating HbAlc data from a single time point to a longer time period; the lack of measurement of HbA1c at similar time points among all participants; the lack of available data on other important risk factors such as microalbuminuria/proteinuria, diet, and medication adherence; and the observational nature of the study in routine primary care practice limit the interpretation of these results. Measurement of serum creatinine has a $\pm 10 \%$ measurement error, which is not corrected by the MDRD equation. Likewise, the MDRD equation may be less precise as an estimate of GFR in patients with relatively preserved renal function. However, given the profound discussions in the literature in recent years about the importance of tight glycemic control, we contend that additional research is needed to better understand potential associations between the magnitude, frequency, and overall time course of fluctuations in HbAlc in routine primary care and the development of CKD and other microvascular complications of diabetes. Additional studies using these or comparable measures to assess patterns of glycemic control in observational studies using primary care patients are needed to determine the validity and reproducibility of this approach. Perhaps the use of one or more of these 10 techniques to better characterize the pattern of glycemic control in large-scale, long-term clinical trials, in which HbA1c measures are routinely obtained at similar and precise intervals, might result in an improved understanding of the relationship with eGFR or other diabetes-specific outcome measures above and beyond those available from conventional analytical techniques.

\section{Conclusion}

These data suggest that traditional demographic and clinical risk factors remain importantly associ- 
ated with changes in eGFR and that fluctuations in glycemic control, though important and in need of further investigation, should be evaluated in the context of the patient's overall risk profile.

\section{References}

1. Effect of intensive therapy on the development and progression of diabetic nephropathy in the Diabetes Control and Complications Trial. The Diabetes Control and Complications (DCCT) Research Group. Kidney Int 1995;47:1703-20.

2. Retinopathy and nephropathy in patients with type 1 diabetes four years after a trial of intensive therapy. The Diabetes Control and Complications Trial/Epidemiology of Diabetes Interventions and Complications Research Group. N Engl J Med 2000;342: 381-9.

3. Intensive blood glucose control with sulphonylureas or insulin compared with conventional treatment and risk of complications in patients with type 2 diabetes (UKPDS 33). UK Prospective Diabetes Study (UKPDS) Group. Lancet 1998;352:837-53.

4. Holman RR, Paul SK, Bethel MA, Matthews DR, Neil HA. 10-year follow-up of intensive glucose control in type 2 diabetes. N Engl J Med 2008;359: 1577-89.

5. Skyler JS. Diabetic complications: the importance of glucose control. Endocrin Metab Clin North Am 1996;25:243-54.

6. Stratton IM, Adler AI, Neil HA, et al. Association of glycaemia with macrovascular and microvascular complications of type 2 diabetes (UKPDS 35): prospective observational study. Br Med J 2000;321: 405-412.

7. Bash LD, Selvin E, Steffes M, Coresh J, Astor BC. Poor glycemic control in diabetes and the risk of incident chronic kidney disease even in the absence of albuminuria and retinopathy. Arch Int Med 2008; 168:2440-7.

8. Ismail-Beigi F, Craven T, Banerji MA, et al. Effect of intensive treatment of hyperglycaemia on $\mathrm{mi}-$ crovascular outcomes in type 2 diabetes: an anal- ysis of the ACCORD randomized trial. Lancet 2010;376:419-30.

9. Lachin JM, Genuth S, Nathan DM, Zinman B, Rutledge BN. Effect of glycemic exposure on the risk of microvascular complications in the diabetes control and complications trial-revisited. Diabetes 2008;57: 995-1001.

10. American Diabetes Association. Standards of Medical Care in Diabetes - 2010. Diabetes Care 2010; 33(Suppl 1):S1-S61.

11. Rigalleau V, Lasseur C, Raffaitin C, et al. Glucose control influences glomerular filtration rate and its prediction in diabetic subjects. Diabetes Care 2006; 29:1491-5.

12. National Kidney Foundation. Glomerular filtration rate (GRF). Available at: http://www.kidney.org/ kidneydisease/ckd/knowGFR.cfm\#chart. Accessed March 2010.

13. Tight blood pressure control and risk of macrovascular and microvascular complications in type 2 diabetes: UKPDS 38. UK Prospective Diabetes Study Group. BMJ 1998;317:703-12.

14. Remuzzi G, Schieppati A, Ruggenenti P. Nephropathy in patients with type 2 diabetes. $\mathrm{N}$ Engl J Med 2002;346:1145-51.

15. Brosnahan G, Fraer M. Chronic kidney disease: whom to screen and how to treat, part 1: definition, epidemiology, and laboratory testing. South Med J 2010;103:140-6.

16. Barit D, Cooper ME. Diabetic patients and kidney protection: an attainable target. J Hypertens 2008; 26:S3-S7.

17. Executive Committee, National Kidney Foundation. Clinical practice recommendation 3: Diabetes and chronic kidney disease in special populations. Am J Kidney Dis 2007;49(2 Suppl 2):S120-30.

18. Hirsch IB, Brownlee M. Beyond hemoglobin a1c need for additional markers of risk for diabetic microvascular complications. JAMA 2010;303:2291-2.

19. Ritz E, Orth SR. Primary care: nephropathy in patients with type 2 diabetes mellitus. N Engl J Med 1999;341:1127-33. 